\title{
Clinical relevance of Lgr5 expression in colorectal cancer patients
}

Young Joo Kim, Dong Hyun Kang, Geum Jong Song, Tae Sung Ahn, Myoung Won Son, Moon Soo Lee, Moo-Jun Baek

Department of Surgery, Soonchunhyang University Cheonan Hospital, Soonchunhyang University College of Medicine, Cheonan, Korea

Purpose: Lgr5 is a well-known stem cell marker in colorectal cancer (CRC). This retrospective study evaluated the expressions of Lgr5 in CRC specimens, and examined whether these expressions were associated with survival outcomes.

Methods: We used immunohistochemistry to retrospectively examine expressions of Lgr5 in paraffin-embedded specimens from 337 patients with CRC between January 2009 and December 2013. All clinicopathologic data were collected by retrospective review based on medical records. The correlation between its expression and clinicopathological data as well as clinical outcomes of patients was analyzed.

Results: Low expression and high expression of Lgr5 in 337 patients were 175 (51.9\%) and 162 (48.1\%), respectively. There was no statistically significant difference in the association of Lgr5 expression with clinicopathologic factors (age, tumor location, lymphatic invasion, vascular invasion, perineural invasion, TNM stage, and differentiation). In the survival analysis, the high expression group of Lgr5 showed a better prognosis than the low expression group in disease-free survival $(P=0.044)$. However, overall survival was not significantly different between the two groups $(\mathrm{P}=0.087)$. In multivariate analysis, we found that high expression of $\mathrm{Lgr} 5$ was independent prognostic factor for tumor relapse (hazard ratio, $0.601 ; 95 \%$ confidence interval, $0.388-0.929 ; \mathrm{P}=0.022$ ).

Conclusion: In present study, high expression of Lgr5 is an independent predictor of favorable prognosis in patients with CRC. So, further well designed, prospective, large scale studies are needed to examine the value of Lgr5 as a prognostic biomarker for CRC.

Keywords: Lgr5, Cancer stem cell, Colorectal neoplasms, Immunohistochemistry, Biomarker

\section{INTRODUCTION}

Colorectal cancer (CRC) is the third most common cancer worldwide, and the third leading cause of cancer death in the Republic of Korea [1,2]. Currently, the treatment of CRC includes surgical resection, adjuvant chemotherapy, radiation therapy, and target therapy, which has recently been used in metastatic CRC. Despite

Received: Jul 31, 2018 Revised: Oct 6, 2018 Accepted: Nov 29, 2018

Correspondence to: Moo-Jun Baek

Department of Surgery, Soonchunhyang University Cheonan Hospital,

31 Suncheonhyang 6-gil, Dongnam-gu, Cheonan 31151, Korea

Tel: +82-41-570-3633, Fax: +82-41-571-0129

E-mail: ssurge@schmc.ac.kr

ORCID: Young Joo Kim (https://orcid.org/0000-0002-4961-9942), Dong Hyun Kang (https://orcid.org/0000-0002-6559-0369), Geum Jong Song (https://orcid.org/00000002-1067-8099), Tae Sung Ahn (https://orcid.org/0000-0001-5617-0365), Myoung Won Son (https://orcid.org/0000-0001-5255-9234), Moon Soo Lee (https://orcid. org/0000-0002-3955-1331), Moo-Jun Baek (https://orcid.org/0000-0003-3567-6687)

Copyright @ 2018 Korean Society of Surgical Oncology

This is an Open Access article distributed under the terms of the Creative Commons Attribution Non-Commercial License (http://creativecommons.org/licenses/by-nc/4.0) which permits unrestricted non-commercial use, distribution, and reproduction in any medium, provided the original work is properly cited. improved survival rates of more than $20 \%$ in the last 20 years owing to these advanced therapies, many patients still experience recurrence, and the overall survival rate is only $65 \%$ to $75 \%[3,4]$. Therefore, it is quite urgent and important to find effective treatments and novel prognostic biomarkers.

Cancer stem cells (CSCs) constitute a small subpopulation of tumor cells that have self-renewing and differentiation abilities, which are properties of normal stem cells. This subpopulation is known to play a crucial role in tumor initiation, metastasis, relapse, and resistance to chemoradiotherapy [5]. In the past, characterizing such CSCs and finding stem cell markers were difficult problems. However, many studies have identified various CSC biomarkers through lineage tracing or organoid culture, and their expression levels and clinical relevance have been reported.

The adenoma-carcinoma sequence, known as the mechanism of tumorigenesis in sporadic CRC, is caused by changes in specific genes. These genetic changes result in mutated adenomatous polyposis coli (APC) or $\beta$-catenin genes in the Wnt pathway, and are known to play an important role in colorectal tumorigenesis $[6,7]$.

Leucine-rich repeat-containing $G$ protein-coupled receptor 5 (Lgr5), a putative stem cell marker in the small intestine and colon, is 
a member of the G protein-coupled receptor (GPCR) family and regulates Wnt signaling to R-spondin receptors [8]. Currently, Lgr5 is the most reliable colorectal CSC marker based on various studies [9].

Overexpression of Lgr5 protein is also reported in hepatocellular cancer, ovarian cancer, basal cell carcinoma, and esophageal cancer. Previous studies have shown that Lgr5 overexpression in $\mathrm{CRC}$ is associated with resistance to chemoradiotherapy, recurrence, and metastasis. Thus, Lgr5 expression may be associated with poor prognosis in CRC, although recent studies found no association with prognosis [10]. Further research is needed to clarify these conflicting results.

Therefore, we investigated the clinical relevance of Lgr5 expression levels, a putative CSC marker, in CRC patients using immunohistochemical staining and medical records.

\section{METHODS}

\section{Patients and tissue specimens}

From January 2009 to December 2013, 417 patients diagnosed with
CRC who underwent complete surgical resection at Soonchunhyang University Cheonan Hospital were enrolled. All specimens were obtained from surgically resected tumors, and the formalin-fixed paraffin-embedded (FFPE) blocks stored in the department of pathology were used. Patients under 18 years of age, who underwent preoperative chemotherapy or radiotherapy, who had unsatisfactory medical records, or whose samples could not be used in immunohistochemistry (IHC) owing to poor preservation of the corresponding FFPE block were excluded. Based on these exclusion criteria, a total of 337 patients were selected for this study. No patient died within 30 days of surgery. All clinicopathological data were collected by retrospective review based on medical records. The survival period was confirmed directly by telephone with the patient or caregiver and used for analysis. Tumor stage was defined according to the TNM classification of the American Joint Committee on International Union against Cancer, 7th edition. The Institutional Review Board of the Soonchunhyang University Cheonan Hospital approved the study (SCHCA NON2017-008-007).

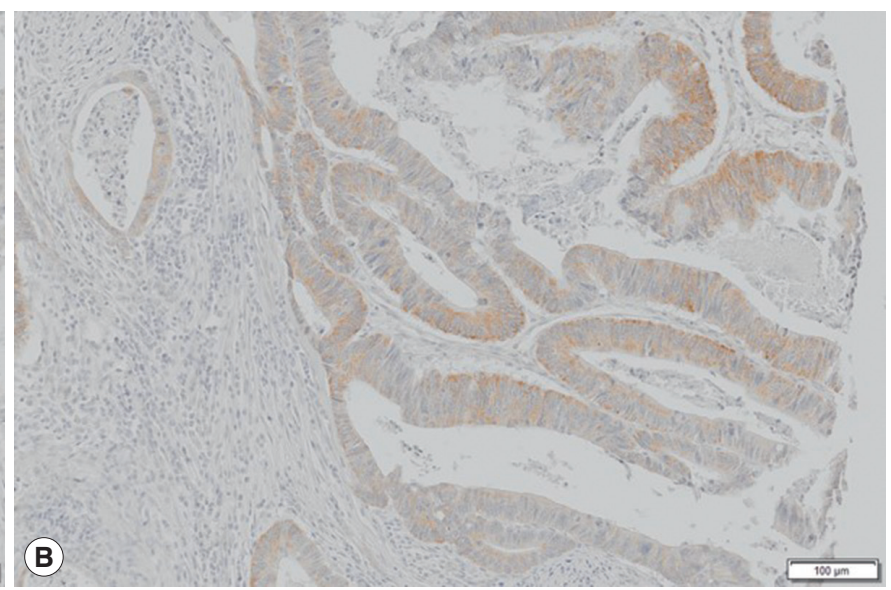

(A)
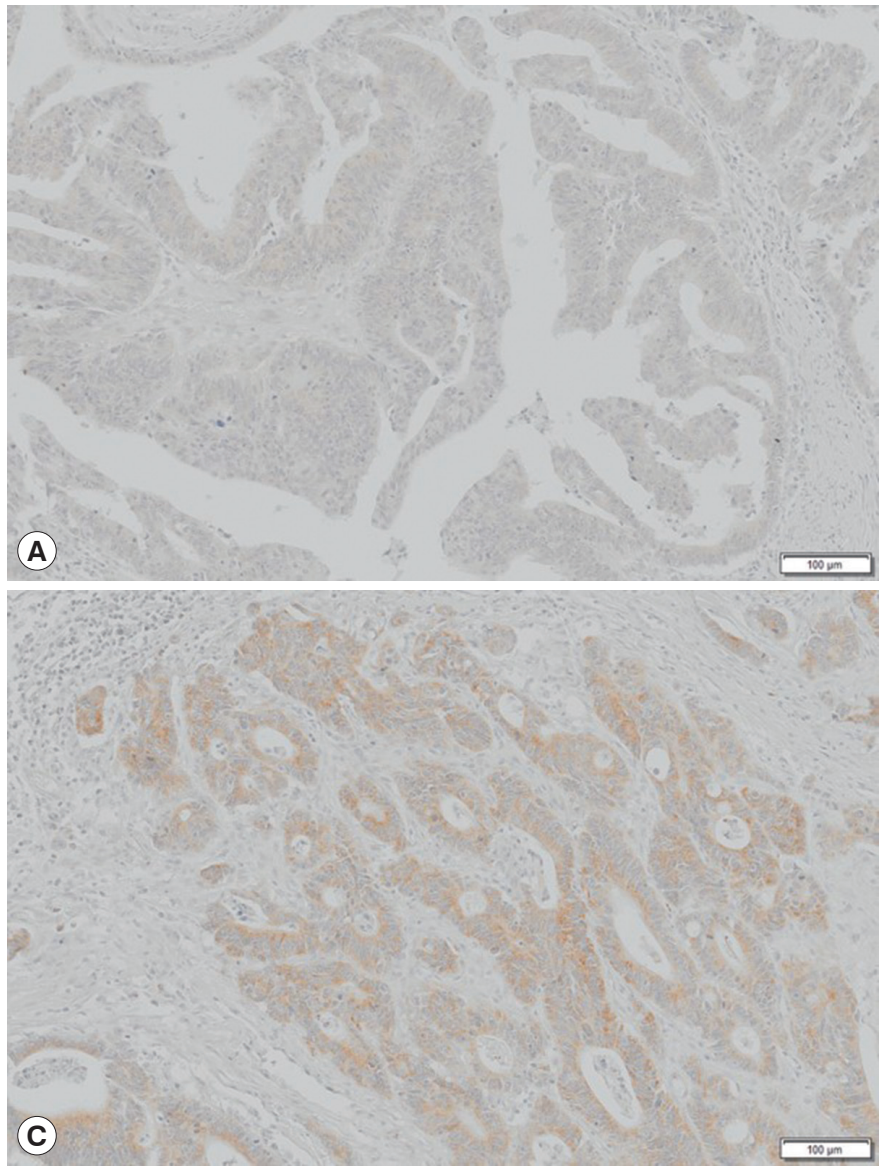

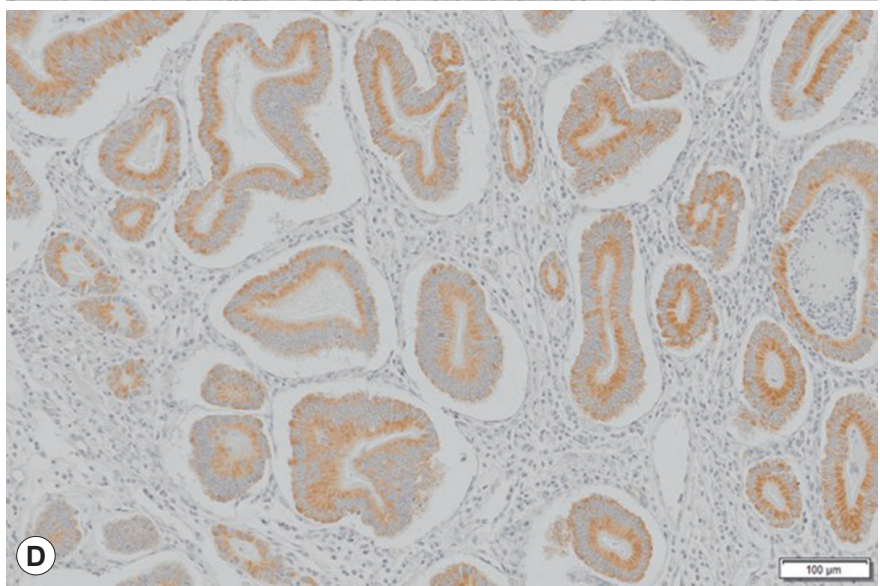

Fig. 1. Differential expression of $\mathrm{Lgr} 5$ in colorectal cancer (CRC) tissues. Immunohistochemistry (IHC) analysis showing Lgr5 in CRC sections. (A) The Lgr5 protein is absent in colorectal cells (staining intensity score 0$)$. (B, C) IHC shows increased expression of Lgr5 protein in CRC cells (scores 1 and 2). (D) Lgr5 is strongly expressed in the cytoplasm of the tumor cells (score 3). Magnification, $\times 100$. 


\section{Immunohistochemistry}

Tissue microarrays (TMA) were fabricated embedding FFPE CRC tissue in a recipient paraffin block using a 2-mm diameter puncher (Unitech Science, Seoul, Korea). The TMA block was sectioned at $4 \mu \mathrm{m}$. TMA slides were deparaffinized and hydrated with xylene and a graded series of alcohols. For antigen retrieval, the TMA slide was boiled in $0.01 \mathrm{M}$ citrate buffer $\mathrm{pH} 6.0$ in a pressure cooker for 15 minutes. The slides were then incubated with $3 \% \mathrm{H}_{2} \mathrm{O}_{2}$ in methanol to inhibit endogenous peroxidase activity. The sections were incubated with anti-Lgr5 antibody (1:100, Abcam, Burlingame, CA, USA) overnight at $4^{\circ} \mathrm{C}$ and then incubated with EnVision HRP-Labeled polymer (Dako, Carpinteria, CA, USA) as secondary antibody. The chromogenic reaction was visualized by treatment with 3,3'-diaminobenzidine (DAB) solution (Dako). Nuclei were counterstained with Harri's hematoxylin solution (Merck, Boston, MA, USA) after the TMA slides were rinsed with distilled water. TMA slides were mounted using Canada balsam (Sigma, Waltham, MA, USA). Protein expression was scored using light microscopy by two independent investigators blind to clinical data, and a consensus score was determined for each specimen. Lgr5 expression levels were evaluated with the IHC score: the percentage of positive cells (percentage score) $\times$ staining intensity (scored as 0 to 3 ). The slides with a final score $\leq 3$ were grouped as low Lgr5 expression, whereas those with score $\geq 4$ were grouped as high Lgr5 expression (Fig. 1).

\section{Statistical analysis}

Statistical analysis was performed using SPSS version 18.0 (SPSS Inc., Chicago, IL, USA) with $\mathrm{P}<0.05$ as the threshold of statistical significance. Chi-square tests were used to analyze the association between Lgr5 expression levels and clinicopathological data. Survival curves were obtained using the Kaplan-Meier survival analysis, and a comparison between the two groups was performed using a log-rank test. Univariate and multivariate analyses of survival rates were performed using the Cox proportional hazard model.

\section{RESULTS}

\section{Relationship between patient characteristics and Lgr5 expression}

Stage I-IV CRC patients were all included (142 females and 195 males). Median age was 64.1 years (range, 29-89 years). According to semi-quantitative analysis of Lgr5 expression, high and low levels were observed in 162 (48.1\%) and 175 patients (51.9\%), respectively. The association of clinicopathological characteristics with Lgr5 expression levels is shown in Table 1. High Lgr5 expression levels showed lower recurrence rate than low expression group
$(\mathrm{P}=0.037)$. However, no statistically significant association was observed with clinicopathologic factors such as age, tumor location, vascular invasion, lymphatic invasion, perineural invasion, pathological TNM stage, or differentiation.

\section{Multivariate and univariate survival analyses using a Cox proportional hazard model in CRC patients}

Univariate analysis showed that vascular invasion $(\mathrm{P}=0.007)$, lymphatic invasion $(\mathrm{P}=0.002)$, perineural invasion $(\mathrm{P}<0.001)$, ad-

Table 1. Association of Lgr5 expression with clinicopathological factors in colorectal cancer patients

\begin{tabular}{|c|c|c|c|c|}
\hline \multirow{2}{*}{ Variable } & \multirow{2}{*}{$\begin{array}{l}\text { Total no. } \\
\text { of cases }\end{array}$} & \multicolumn{2}{|c|}{ Lgr5 expression (\%) } & \multirow{2}{*}{ P-value } \\
\hline & & Low & High & \\
\hline Total & 337 (100) & $175(51.9)$ & $162(48.1)$ & \\
\hline Age (yr) & & & & 0.401 \\
\hline$<60$ & $120(35.6)$ & $66(37.7)$ & 54 (33.3) & \\
\hline$\geq 60$ & $217(64.4)$ & 109 (62.3) & $108(66.7)$ & \\
\hline Tumor location & & & & 0.861 \\
\hline Right & $116(34.4)$ & $61(34.9)$ & $55(34.0)$ & \\
\hline Left & $221(65.6)$ & $114(65.1)$ & 107 (66.0) & \\
\hline Vascular invasion & & & & 0.561 \\
\hline Yes & 54 (16.0) & $30(17.1)$ & $24(14.8)$ & \\
\hline No & $283(84.0)$ & $145(82.9)$ & 138 (85.2) & \\
\hline Lymphatic invasion & & & & 0.936 \\
\hline Yes & 95 (28.2) & $49(28.0)$ & $46(28.4)$ & \\
\hline No & $242(71.8)$ & $126(72.0)$ & $116(71.6)$ & \\
\hline Perineural invasion & & & & 0.963 \\
\hline Yes & 114 (33.8) & $59(33.7)$ & $55(34.0)$ & \\
\hline No & $223(66.2)$ & 116 (66.3) & $107(66.0)$ & \\
\hline T stage & & & & 0.649 \\
\hline $\mathrm{T} 1$ & $26(7.7)$ & $11(6.8)$ & $15(8.6)$ & \\
\hline T2 & $44(13.1)$ & $17(10.5)$ & 27 (15.4) & \\
\hline $\mathrm{T} 3$ & $213(63.2)$ & $112(69.1)$ & $101(57.7)$ & \\
\hline T4 & 54 (16.0) & $22(13.6)$ & 32 (18.3) & \\
\hline N stage & & & & 0.426 \\
\hline NO & $214(63.5)$ & $99(61.1)$ & $115(65.7)$ & \\
\hline N1 & $80(23.7)$ & $41(25.3)$ & 39 (22.3) & \\
\hline N2 & $43(12.8)$ & $22(13.6)$ & $21(12.0)$ & \\
\hline Distant metastasis & & & & 0.966 \\
\hline Absent & 316 (93.8) & 152 (93.8) & 164 (93.7) & \\
\hline Present & $21(6.2)$ & $10(6.2)$ & $11(6.3)$ & \\
\hline Differentiation & & & & 0.621 \\
\hline Well/moderate & 316 (93.8) & 163 (93.1) & $153(94.4)$ & \\
\hline Poorly/undifferentiated & $21(6.2)$ & $12(6.9)$ & $9(5.6)$ & \\
\hline Stage & & & & 0.730 \\
\hline$|\&| \mid$ & $205(60.8)$ & $108(61.7)$ & 97 (59.9) & \\
\hline III\&IV & $132(39.2)$ & $67(38.3)$ & $65(40.1)$ & \\
\hline Recurrence & & & & 0.037 \\
\hline$(-)$ & $251(74.5)$ & $122(69.7)$ & $129(79.6)$ & \\
\hline$(+)$ & $86(25.5)$ & 53 (30.3) & 33 (20.4) & \\
\hline
\end{tabular}

Values are presented as number (\%). 
vanced stage $(\mathrm{P}<0.001)$, and low Lgr5 expression $(\mathrm{P}=0.045)$ were independent negative prognostic factors for disease-free survival. In addition, lymphatic invasion $(\mathrm{P}=0.002)$, perineural invasion $(\mathrm{P}<0.001)$, and advanced stage $(\mathrm{P}=0.001)$ were independent prognostic factors for overall survival, whereas vascular invasion $(\mathrm{P}=0.108)$ and Lgr5 expression levels $(\mathrm{P}=0.093)$ did not show sta- tistical significance. Age, sex, and tumor location were also not associated with survival outcomes (Table 2). We also performed multivariate analysis with significant factors in univariate analysis. Multivariate analysis showed that low Lgr5 expression was an independent prognostic factor for tumor recurrence (hazard ratio [HR], 0.601; 95\% confidence interval [CI], 0.388-0.929; P = 0.022),

Table 2. Univariate analysis of overall survival and disease-free survival in colorectal cancer patients

\begin{tabular}{|c|c|c|c|c|}
\hline \multirow{2}{*}{ Variable } & \multicolumn{2}{|c|}{ OS } & \multicolumn{2}{|c|}{ DFS } \\
\hline & $\mathrm{HR}(95 \% \mathrm{Cl})$ & P-value & $\mathrm{HR}(95 \% \mathrm{Cl})$ & P-value \\
\hline Age (< 60 yr vs. $\geq 60$ yr) & $1.589(0.727-3.475)$ & 0.246 & $1.519(0.985-2.408)$ & 0.076 \\
\hline Sex (male vs. female) & $1.212(0.592-2.484)$ & 0.599 & $1.112(0.727-1.700)$ & 0.625 \\
\hline Tumor location (right vs. left) & 1.137 (0.521-2.483) & 0.747 & $0.945(0.607-1.473)$ & 0.804 \\
\hline Vascular invasion (yes vs. no) & $2.006(0.859-4.685)$ & 0.108 & $2.003(1.214-3.306)$ & 0.007 \\
\hline Lymphatic invasion (yes vs. no) & $3.035(1.483-6.214)$ & 0.002 & $1.973(1.276-3.048)$ & 0.002 \\
\hline Perineural invasion (yes vs. no) & $4.084(1.938-8.606)$ & $<0.001$ & $3.279(2.135-5.035)$ & $<0.001$ \\
\hline Stage (III\&IV vs. I\&II) & $3.675(1.718-7.861)$ & 0.001 & $3.171(2.051-4.904)$ & $<0.001$ \\
\hline Lgr5 expression (high vs. low) & $0.521(0.244-1.115)$ & 0.093 & $0.643(0.417-0.994)$ & 0.045 \\
\hline
\end{tabular}

OS, overall survival; DFS, disease-free survival; $\mathrm{HR}$, hazard ratio; $\mathrm{Cl}$, confidence interval.

Table 3. Multivariate analysis of overall survival and disease-free survival in colorectal cancer patients

\begin{tabular}{lcccccc}
\hline \multirow{2}{*}{ Variable } & \multicolumn{2}{c}{ OS } & & & DFS \\
\cline { 2 - 3 } \cline { 5 - 6 } & HR (95\% Cl) & P-value & HR (95\% Cl) & P-value \\
\hline Vascular invasion (yes vs. no) & $1.122(0.456-2.759)$ & 0.802 & & $1.382(0.806-2.372)$ & 0.240 \\
Lymphatic invasion (yes vs. no) & $1.285(0.527-3.131)$ & 0.582 & & $0.770(0.452-1.312)$ & 0.336 \\
Perineural invasion (yes vs. no) & $2.936(1.326-6.499)$ & 0.008 & & $2.519(1.599-3.967)$ & $<0.001$ \\
Stage (III\&IV vs. I\&II) & $2.531(1.123-5.704)$ & 0.025 & & $2.398(1.513-3.802)$ & $<0.001$ \\
Lgr5 expression (high vs. low) & $0.501(0.234-1.073)$ & 0.075 & & $0.601(0.388-0.929)$ & 0.022 &
\end{tabular}

OS, overall survival; DFS, disease-free survival; $\mathrm{HR}$, hazard ratio; $\mathrm{Cl}$, confidence interval.
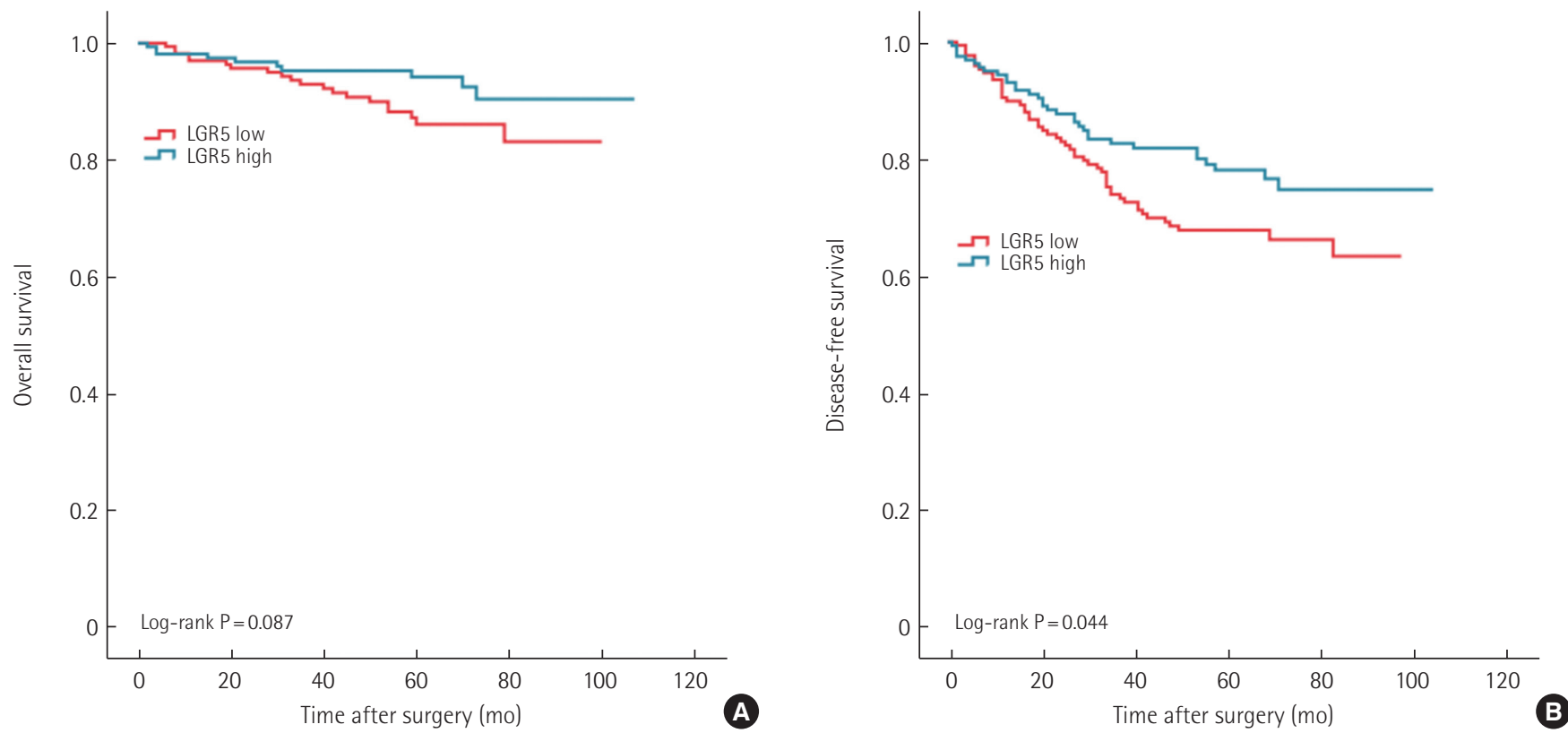

Fig. 2. Kaplan-Meier curves for overall survival rates (A) and disease-free survival rates (B) according to Lgr5 expression (high vs. low). 
but not for overall survival (HR, 0.501; 95\% CI, 0.234-1.073; $\mathrm{P}=0.075$ ) (Table 3).

Kaplan-Meier analysis and log-rank test showed that the high Lgr5 expression group (mean of $100.8 \pm 1.8$ months) did not show a statistically significant difference in survival rates compared with the low Lgr5 expression group (mean of $90.3 \pm 2.0$ months, $\mathrm{P}=0.087$ (Fig. 2A). The recurrence rate was also lower in the high than in the low Lgr5 expression group $(\mathrm{P}=0.044)$ (Fig. 2B).

\section{DISCUSSION}

In 1997, CSCs were first isolated from patients with acute myeloid leukemia, and for the first time from solid tumors in patients with breast cancer [11,12]. Subsequently, CSCs have been isolated from various solid tumors such as brain tumor, pancreatic cancer, melanoma, and CRC [13-16]. CSCs are side populations of tumor cells and are well known to be the cause of tumor initiation, proliferation, and metastasis.

Barker et al. [17] showed that Lgr5 expressing in the crypt base columnar cell is a potent stem cell marker in the small intestine and colon by lineage tracing experiments in mice with knock-in Lgr5-EGFP-IRES-creERT2. The authors also demonstrated that Lgr5 expressing in colon and small intestinal stem cells (ISCs) induce intestinal neoplasia in APC-truncated mice with adenoma, showing that Lgr5 is a reliable CRC stem cell marker [9].

In various studies, Lgr5+ CSCs are mainly located at the crypt base in the developmental stage of the tumor, and then migrate upward in the crypt while showing a patched distribution in advanced cancer. In the present study, Lgr5 was observed in a similar distribution, and we found that Lgr5 levels were higher in the gland than in the stroma in advanced colon cancer. In addition, high Lgr5 expression levels were found in $48.1 \%$ of all patients, similar to what has been previously reported.

The role of Lgr5 and its clinical implications have been reported in various studies, but the issue is still controversial. In the present study, no association was found between Lgr5 expression levels and clinicopathological factors, although Lgr5 expression in survival analysis showed favorable results in recurrence. Univariate and multivariate analyses of survival also showed Lgr5 expression as an independent factor in tumor relapse. In addition, although not statistically significant, the association with the overall survival rate also tended to be favorable. Consistent with these results, de Sousa E Melo et al. [18] showed that high expression levels of Wnt-targeted genes, which comprise ASCL2, Lgr5, AXIN2, and APCDD1, are associated with a favorable prognosis in CRC patients. These results are consistent with the low expression levels of these ISC markers resulting from methylation of the $\mathrm{CpG}$ island promoter, which has been reported as a predictor of tumor relapse. Walker et al. [19] also investigated the role of Lgr5 using small interfering RNA-mediated knockdown of its gene in CRC cell lines, which resulted in loss of cell-cell adhesion, a tendency to the mesenchymal phenotype, enhanced clonogenicity, and upregulation of a variety of epithelial to mesenchymal transition (EMT) pathway genes.

Wnt, Notch, hedgehog, epidermal growth factor receptor (EGFR), and EMT are important signaling pathways in maintaining homeostasis and carcinogenesis in CRC. Among these, activation of the Wnt signaling pathway by APC or mutations in $\beta$-catenin is the most prominent in the adenoma-carcinoma sequence. However, Lgr 5 reduces transcription of Wnt-responsive genes by inhibiting the Wnt signaling pathway. Thus, it is hypothesized that this inhibition may be related to the association between high Lgr5 expression levels and favorable prognosis.

Lgr5 expression levels also show a positive relation with Ki-67, which is expressed as a nuclear antigen in proliferating cells in the G1 to M phase transition. Melling et al. [20] reported improved survival associated with high Ki-67 expression levels in 1,653 CRC patients, and showed that Ki-67 is an independent prognostic factor for survival through multivariable Cox proportional regression. In addition, the proliferative activity of these tumors is negatively related to aggressiveness and metastasis in CRC, which indirectly suggests that high expression levels of Lgr5 may have a favorable prognosis [21].

These results, however, are in contrast to CSCs features such as resistance to chemoradiotherapy, cancer progression, or metastasis. In fact, overexpression of Lgr5 is associated with poor prognosis and other high-risk factors in CRC patients.

According to recently published meta-analysis, Lgr5 expression levels in CRC is a poor prognostic factor in survival and is associated with advanced stage [22,23]. Merlos-Suarez et al. [24] have shown that EphB2, an ISC marker, is more abundant in the intestinal crypt base than in the villus. The authors also showed that the group with high expression of EphB2-derived ISC signatures, including Lgr5 in CRC, is significantly associated with tumor recurrence. These negative correlations between Lgr5 expression levels and survival outcome was reported not only in CRC but also in other solid tumors [25,26].

In contrast, Ziskin et al. [10] reported a lack of significant association between Lgr5 expression levels and 5-year overall survival in 891 patients with CRC. These reports are inconsistent with our results, and the studies are difficult to compare directly owing to its heterogeneity in experimental conditions.

Recent studies have reported the role of Lgr5 in CRC. In an animal model using AKVPL organoid, primary tumors were not 
completely removed with ablation of Lgr5+ CSCs and rapidly regrew after cessation of treatment, whereas metastatic lesions were completely eliminated [27]. These results are consistent with other studies, and result from the plasticity of CSCs and the maintenance of the CSCs pool by Lgr5- cells in primary tumors [27-29]. Fujii and Sato [28] reported significant volume regression after induction of Lgr5+ CSCs through anti-EGFR treatment following Lgr5+ CSC ablation in vitro. However, the mechanisms underlying differences in treatment response between primary tumors and metastatic lesions remain unknown. Therefore, to clarify the role of Lgr5+ CSCs, how metastasis is induced in these cells and how they are affected by the tumor microenvironment need to be investigated.

Lgr5+ CSCs clearly play a critical role in colorectal carcinogenesis. Thus, Lgr5+ CSCs are assumed to be associated with clinicopathological parameters, but no association was found in our study. Therefore, it is difficult to explain the relationship between high Lgr5 expression levels and favorable outcome in this study, but overexpression of Lgr 5 may be associated with other prognostic factors or signaling pathways. Some studies have reported an association between Lgr5 expression and BRAF or microsatellite instability status (not included in this study), which are prognostic factors of CRC $[10,30]$. Therefore, correlation analysis with various prognostic factors is needed to clarify the role of Lgr5 as a prognostic biomarker and to uncover the mechanisms underlying our results.

There are various limitations in our study. Firstly, we selected a single marker, and did not investigate various colorectal CSCs signatures. Several studies have identified CSC markers (e.g., CD133, CD44, CD166, and ALDH1), and CD133, a cell surface marker, has been shown to be an independent predictor of unfavorable prognosis. Secondly, retrospective studies and results from a single institution may be less reliable than multicenter prospective studies. Thirdly, this study may be biased in survival outcome because patients did not consider other treatment strategies or chemotherapy regimens. Finally, there is still no consensus for reference Lgr5 expression levels, and therefore different cutoff values are used in each study, consequently reaching inconsistent results.

In conclusion, our study demonstrated a positive correlation between Lgr5 overexpression and tumor recurrence in patients with CRC. We also showed that Lgr5 expression is an independent prognostic factor for recurrence. However, to clarify these results, multicenter prospective studies and well-designed functional studies will be needed. Through these studies, we expect Lgr5 to be a valuable prognostic biomarker.

\section{CONFLICT OF INTEREST}

MJB, an editor-in-chief of Korean Journal of Clinical Oncology, is the corresponding author of this article. However, he played no role whatsoever in the editorial evaluation of this article or the decision to publish it. Except for that, no potential conflict of interest relevant to this article was reported.

\section{ACKNOWLEDGMENTS}

This research was supported by a grant of the Korea Health Technology R\&D Project through the Korea Health Industry Development Institute (KHID), funded by the Ministry of Health \& Welfare, Republic of Korea (grant number: HI17C0031).

\section{REFERENCES}

1. Bray F, Ferlay J, Soerjomataram I, Siegel RL, Torre LA, Jemal A. Global cancer statistics 2018: GLOBOCAN estimates of incidence and mortality worldwide for 36 cancers in 185 countries. CA Cancer J Clin 2018;68:394-424.

2. National Cancer Information Center. Caner statistics, 2017 [Internet]. Goyang (KR): National Cancer Information Center [cited 2018 Jul 10]. Available from: https://www.cancer.go.kr/lay1/S1T645C646/ contents.do

3. Jung KW, Won YJ, Oh CM, Kong HJ, Lee DH, Lee KH, et al. Cancer statistics in Korea: incidence, mortality, survival, and prevalence in 2014. Cancer Res Treat 2017;49:292-305.

4. Siegel RL, Miller KD, Jemal A. Cancer statistics, 2018. CA Cancer J Clin 2018:68:7-30.

5. Ricci-Vitiani L, Lombardi DG, Pilozzi E, Biffoni M, Todaro M, Peschle C, et al. Identification and expansion of human colon-cancer-initiating cells. Nature 2007;445:111-5.

6. Morin PJ, Sparks AB, Korinek V, Barker N, Clevers H, Vogelstein B, et al. Activation of beta-catenin-Tcf signaling in colon cancer by mutations in beta-catenin or APC. Science 1997;275:1787-90.

7. Powell SM, Zilz N, Beazer-Barclay Y, Bryan TM, Hamilton SR, Thibodeau SN, et al. APC mutations occur early during colorectal tumorigenesis. Nature 1992;359:235-7.

8. de Lau W, Peng WC, Gros P, Clevers H. The R-spondin/Lgr5/ Rnf43 module: regulator of Wnt signal strength. Genes Dev 2014; 28:305-16.

9. Barker N, Ridgway RA, van Es JH, van de Wetering M, Begthel H, van den Born M, et al. Crypt stem cells as the cells-of-origin of intestinal cancer. Nature 2009;457:608-11.

10. Ziskin JL, Dunlap D, Yaylaoglu M, Fodor IK, Forrest WF, Patel R, et al. In situ validation of an intestinal stem cell signature in colorectal cancer. Gut 2013;62:1012-23.

11. Al-Hajj M, Wicha MS, Benito-Hernandez A, Morrison S, Clarke MF. Prospective identification of tumorigenic breast cancer cells. 
Proc Natl Acad Sci U S A 2003;100:3983-8.

12. Bonnet D, Dick JE. Human acute myeloid leukemia is organized as a hierarchy that originates from a primitive hematopoietic cell. Nat Med 1997;3:730-7.

13. Schatton T, Murphy GF, Frank NY, Yamaura K, Waaga-Gasser AM, Gasser M, et al. Identification of cells initiating human melanomas. Nature 2008;451:345-9.

14. Li C, Heidt DG, Dalerba P, Burant CF, Zhang L, Adsay V, et al. Identification of pancreatic cancer stem cells. Cancer Res 2007;67: 1030-7.

15. O'Brien CA, Pollett A, Gallinger S, Dick JE. A human colon cancer cell capable of initiating tumour growth in immunodeficient mice. Nature 2007;445:106-10.

16. Singh SK, Hawkins C, Clarke ID, Squire JA, Bayani J, Hide T, et al. Identification of human brain tumour initiating cells. Nature 2004; 432:396-401.

17. Barker N, van Es JH, Kuipers J, Kujala P, van den Born M, Cozijnsen $\mathrm{M}$, et al. Identification of stem cells in small intestine and colon by marker gene Lgr5. Nature 2007;449:1003-7.

18. de Sousa E Melo F, Colak S, Buikhuisen J, Koster J, Cameron K, de Jong JH, et al. Methylation of cancer-stem-cell-associated Wnt target genes predicts poor prognosis in colorectal cancer patients. Cell Stem Cell 2011;9:476-85.

19. Walker F, Zhang HH, Odorizzi A, Burgess AW. LGR5 is a negative regulator of tumourigenicity, antagonizes Wnt signalling and regulates cell adhesion in colorectal cancer cell lines. PLoS One 2011;6: e22733.

20. Melling N, Kowitz CM, Simon R, Bokemeyer C, Terracciano L, Sauter G, et al. High Ki67 expression is an independent good prognostic marker in colorectal cancer. J Clin Pathol 2016;69:209-14.

21. Anjomshoaa A, Nasri S, Humar B, McCall JL, Chatterjee A, Yoon HS, et al. Slow proliferation as a biological feature of colorectal can- cer metastasis. Br J Cancer 2009; 101:822-8.

22. Yang L, Lin Y, Liu Z, Li Y, Liu Y, Liang R. Impact of LGR5 in colorectal cancer on overall and progression-free survival: a systematic review and meta-analysis. Int J Clin Exp Med 2016;9:10537-43.

23. Jiang Y, Li W, He X, Zhang H, Jiang F, Chen Z. Lgr5 expression is a valuable prognostic factor for colorectal cancer: evidence from a meta-analysis. BMC Cancer 2015;15:948.

24. Merlos-Suarez A, Barriga FM, Jung P, Iglesias M, Cespedes MV, Rossell D, et al. The intestinal stem cell signature identifies colorectal cancer stem cells and predicts disease relapse. Cell Stem Cell 2011;8:511-24.

25. Yang L, Tang H, Kong Y, Xie X, Chen J, Song C, et al. LGR5 promotes breast cancer progression and maintains stem-like cells through activation of Wnt/beta-catenin signaling. Stem Cells 2015; 33:2913-24.

26. Simon E, Petke D, Boger C, Behrens HM, Warneke V, Ebert M, et al. The spatial distribution of LGR5+ cells correlates with gastric cancer progression. PLoS One 2012;7:e35486.

27. de Sousa e Melo F, Kurtova AV, Harnoss JM, Kljavin N, Hoeck JD, Hung J, et al. A distinct role for Lgr5(+) stem cells in primary and metastatic colon cancer. Nature 2017;543:676-80.

28. Fujii M, Sato T. Defining the role of Lgr5(+) stem cells in colorectal cancer: from basic research to clinical applications. Genome Med 2017;9:66.

29. Junttila MR, Mao W, Wang X, Wang BE, Pham T, Flygare J, et al. Targeting LGR5+ cells with an antibody-drug conjugate for the treatment of colon cancer. Sci Transl Med 2015;7:314ra186.

30. Hutchins G, Southward K, Handley K, Magill L, Beaumont C, Stahlschmidt J, et al. Value of mismatch repair, KRAS, and BRAF mutations in predicting recurrence and benefits from chemotherapy in colorectal cancer. J Clin Oncol 2011;29:1261-70. 